\title{
MILAGRO OBSERVATIONS OF MULTI-TeV EMISSION FROM GALACTIC SOURCES IN THE FERMI BRIGHT SOURCE LIST
}

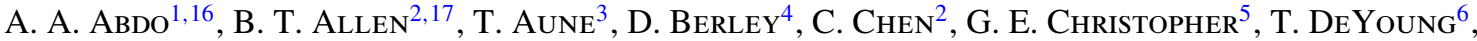 \\ B. L. Dingus ${ }^{7}$, R. W. Ellsworth ${ }^{8}$, M. M. Gonzalez ${ }^{9}$, J. A. Goodman ${ }^{4}$, E. Hays ${ }^{10}$, C. M. Hoffman ${ }^{7}$, \\ P. H. Hüntemeyer ${ }^{11}$, B. E. Kolterman ${ }^{5}$, J. T. Linnemann ${ }^{1}$, J. E. McEnery ${ }^{10}$, T. Morgan ${ }^{12}$, A. I. Mincer $^{5}$, \\ P. Nemethy ${ }^{5}$, J. Pretz ${ }^{7}$, J. M. Ryan ${ }^{12}$, P. M. Saz Parkinson ${ }^{3}$, A. Shoup ${ }^{13}$, G. Sinnis ${ }^{7}$, A. J. Smith ${ }^{4}$, \\ V. VAsileiou ${ }^{4,14}$, G. P. Walker ${ }^{7,18}$, D. A. Williams ${ }^{3}$, and G. B. YodH $^{2}$ \\ ${ }^{1}$ Department of Physics and Astronomy, Michigan State University, 3245 BioMedical Physical Sciences Building, East Lansing, MI 48824, USA \\ 2 Department of Physics and Astronomy, University of California, Irvine, CA 92697, USA \\ 3 Santa Cruz Institute for Particle Physics, University of California, 1156 High Street, Santa Cruz, CA 95064, USA \\ 4 Department of Physics, University of Maryland, College Park, MD 20742, USA \\ 5 Department of Physics, New York University, 4 Washington Place, New York, NY 10003, USA \\ 6 Department of Physics, Pennsylvania State University, University Park, PA 16802, USA \\ 7 Group P-23, Los Alamos National Laboratory, P.O. Box 1663, Los Alamos, NM 87545, USA \\ 8 Department of Physics and Astronomy, George Mason University, 4400 University Drive, Fairfax, VA 22030, USA \\ 9 Instituto de Astronomía, Universidad Nacional Autónoma de México, 04510 México, D.F., Mexico \\ 10 NASA Goddard Space Flight Center, Greenbelt, MD 20771, USA \\ 11 Department of Physics, University of Utah, Salt Lake City, UT 84112, USA \\ 12 Department of Physics, University of New Hampshire, Morse Hall, Durham, NH 03824, USA \\ 13 Ohio State University, Lima, OH 45804, USA \\ 14 CRESST NASA/Goddard Space Flight Center, MD 20771, USA \\ 15 University of Maryland, Baltimore County, MD 21250, USA \\ Received 2009 April 6; accepted 2009 June 30; published 2009 July 13
}

\begin{abstract}
We present the result of a search of the Milagro sky map for spatial correlations with sources from a subset of the recent Fermi Bright Source List (BSL). The BSL consists of the 205 most significant sources detected above $100 \mathrm{MeV}$ by the Fermi Large Area Telescope. We select sources based on their categorization in the BSL, taking all confirmed or possible Galactic sources in the field of view of Milagro. Of the 34 Fermi sources selected, 14 are observed by Milagro at a significance of 3 standard deviations or more. We conduct this search with a new analysis which employs newly optimized gamma-hadron separation and utilizes the full eight-year Milagro data set. Milagro is sensitive to gamma rays with energy from 1 to $100 \mathrm{TeV}$ with a peak sensitivity from 10 to $50 \mathrm{TeV}$ depending on the source spectrum and declination. These results extend the observation of these sources far above the Fermi energy band. With the new analysis and additional data, multi-TeV emission is definitively observed associated with the Fermi pulsar, J2229.0+6114, in the Boomerang pulsar wind nebula (PWN). Furthermore, an extended region of multi-TeV emission is associated with the Fermi pulsar, J0634.0+1745, the Geminga pulsar.
\end{abstract}

Key words: gamma rays: observations - pulsars: general - supernova remnants

\section{INTRODUCTION}

The Milagro gamma-ray observatory has performed the most sensitive survey of 1-100 TeV gamma rays from the Northern Hemisphere sky (Abdo et al. 2007a, 2007b). The Milagro data set is ideal for searching for new classes of gamma-ray sources. The recent release of the Bright Source List (BSL) by the Fermi collaboration (Abdo et al. 2009) presents such an opportunity by looking for coincidences of $>1 \mathrm{TeV}$ emission with these $\mathrm{GeV}$ sources. There are 34 sources in the BSL within Milagro's field of view that are not associated with extragalactic sources. We present a search of the Milagro data for excesses between 1 and $100 \mathrm{TeV}$ coincident with these 34 potential Galactic sources. The analysis presented here differs from previous analyses (Abdo et al. 2007a, 2007b, 2008) by optimizing the event weighting and Gaussian weighting separately in bins of event size (measured by the fraction of channels hit in the instrument).

\footnotetext{
16 Current address: Space Science Division, Naval Research Laboratory, Washington, DC 20375, USA.

17 Current address: Harvard-Smithsonian Center for Astrophysics, Cambridge, MA 02138, USA.

18 Current address: National Security Technologies, Las Vegas, NV 89102, USA.
}

With the improved analysis and an additional year and a half of data, the sensitivity has increased by $15 \%-25 \%$, depending on the spectrum of the source.

\section{ANALYSIS AND RESULTS}

We select Fermi-LAT sources in the field of view of Milagro (with declination $\delta>-5^{\circ}$ ) based on their categorization in the BSL. Sources are selected which are confirmed or potential Galactic sources. Sources that are identified as extragalactic are omitted. Sixteen of the selected sources were categorized in the BSL as confirmed pulsars (PSR) and one is a high-mass X-ray binary $(\mathrm{HXB})$. Five sources have a potential association with a supernova remnant (SNR), and 12 have no clear association. For each of these 34 sources, we calculate the statistical significance of the Milagro data at the BSL position and estimate the flux or flux limit under the assumption that the emission is from a single point source.

The flux measurements given in Table 1 are derived with a similar approach to Abdo et al. (2007b). The flux is measured with an assumed spectrum of $E^{-2.6}$ without a cutoff. The dependence of the calculated flux on the true spectrum is minimized when the flux is quoted at the median energy of 
Table 1

Summary of the Search for TeV Emission from Sources in the Fermi BSL

\begin{tabular}{|c|c|c|c|c|c|c|c|c|}
\hline $\begin{array}{l}\text { Name } \\
(\text { (OFGL) }\end{array}$ & Type & $\begin{array}{l}\text { R.A. } \\
\text { (deg) }\end{array}$ & $\begin{array}{l}\text { Decl. } \\
\text { (deg) }\end{array}$ & $\begin{array}{c}l \\
(\mathrm{deg})\end{array}$ & $\begin{array}{c}b \\
(\mathrm{deg})\end{array}$ & $\begin{array}{c}\text { Flux } \\
\left(\times 10^{-17} \mathrm{TeV}^{-1}\right. \\
\left.\mathrm{s}^{-1} \mathrm{~cm}^{-2}\right)\end{array}$ & $\begin{array}{l}\text { Signif. } \\
(\sigma \text { 's })\end{array}$ & $\begin{array}{l}\mathrm{TeV} \\
\text { Assoc. }\end{array}$ \\
\hline $\mathrm{J} 0007.4+7303$ & PSR & 1.85 & 73.06 & 119.69 & 10.47 & $<90.4$ & 2.6 & \\
\hline $\mathrm{J} 0030.3+0450$ & PSR & 7.60 & 4.85 & 113.11 & -57.62 & $<20.9$ & -1.7 & \\
\hline $\mathrm{J} 0240.3+6113$ & HXB & 40.09 & 61.23 & 135.66 & 1.07 & $<26.2$ & 0.7 & LSI +61 303 \\
\hline $\mathrm{J} 0357.5+3205$ & PSR & 59.39 & 32.08 & 162.71 & -16.06 & $<16.5$ & -0.1 & \\
\hline J0534.6+2201 & PSR & 83.65 & 22.02 & 184.56 & -5.76 & $162.6 \pm 9.4$ & 17.2 & Crab \\
\hline J0613.9-0202 & PSR & 93.48 & -2.05 & 210.47 & -9.27 & $<60.0$ & -0.0 & \\
\hline J0617.4+2234 & $\mathrm{SNR}^{\mathrm{a}}$ & 94.36 & 22.57 & 189.08 & 3.07 & $28.8 \pm 9.5$ & 3.0 & IC443 \\
\hline J0631.8+1034 & PSR & 97.95 & 10.57 & 201.30 & 0.51 & $47.2 \pm 12.9$ & 3.7 & \\
\hline J0633.5+0634 & PSR & 98.39 & 6.58 & 205.04 & -0.96 & $<50.2$ & 1.4 & \\
\hline $\mathrm{J} 0634.0+1745$ & PSR & 98.50 & 17.76 & 195.16 & 4.29 & $37.7 \pm 10.7$ & 3.5 & $\begin{array}{l}\text { MGRO C3 } \\
\text { Geminga }\end{array}$ \\
\hline J0643.2+0858 & & 100.82 & 8.98 & 204.01 & 2.29 & $<30.5$ & 0.3 & \\
\hline J1653.4-0200 & & 253.35 & -2.01 & 16.55 & 24.96 & $<51.0$ & -0.5 & \\
\hline $\mathrm{J} 1830.3+0617$ & & 277.58 & 6.29 & 36.16 & 7.54 & $<32.8$ & 0.2 & \\
\hline $\mathrm{J} 1836.2+5924$ & PSR & 279.06 & 59.41 & 88.86 & 25.00 & $<14.6$ & -0.9 & \\
\hline J1844.1-0335 & & 281.04 & -3.59 & 28.91 & -0.02 & $148.4 \pm 34.2$ & 4.3 & \\
\hline J1848.6-0138 & & 282.16 & -1.64 & 31.15 & -0.12 & $<91.7$ & 1.7 & \\
\hline $\mathrm{J} 1855.9+0126$ & $\mathrm{SNR}^{\mathrm{a}}$ & 283.99 & 1.44 & 34.72 & -0.35 & $<89.5$ & 2.2 & \\
\hline $\mathrm{J} 1900.0+0356$ & & 285.01 & 3.95 & 37.42 & -0.11 & $70.7 \pm 19.5$ & 3.6 & \\
\hline $\mathrm{J} 1907.5+0602$ & PSR & 286.89 & 6.03 & 40.14 & -0.82 & $116.7 \pm 15.8$ & 7.4 & $\begin{array}{c}\text { MGRO J1908+06 } \\
\text { H.E.S.S. J1908+063 }\end{array}$ \\
\hline $\mathrm{J} 1911.0+0905$ & $\mathrm{SNR}^{\mathrm{a}}$ & 287.76 & 9.09 & 43.25 & -0.18 & $<41.7$ & 1.5 & \\
\hline $\mathrm{J} 1923.0+1411$ & $\mathrm{SNR}^{\mathrm{a}}$ & 290.77 & 14.19 & 49.13 & -0.40 & $39.4 \pm 11.5$ & 3.4 & H.E.S.S. J1923+141 \\
\hline $\mathrm{J} 1953.2+3249$ & PSR & 298.32 & 32.82 & 68.75 & 2.73 & $<17.0$ & 0.0 & \\
\hline $\mathrm{J} 1954.4+2838$ & $\mathrm{SNR}^{\mathrm{a}}$ & 298.61 & 28.65 & 65.30 & 0.38 & $37.1 \pm 8.6$ & 4.3 & \\
\hline $\mathrm{J} 1958.1+2848$ & PSR & 299.53 & 28.80 & 65.85 & -0.23 & $34.7 \pm 8.6$ & 4.0 & \\
\hline $\mathrm{J} 2001.0+4352$ & & 300.27 & 43.87 & 79.05 & 7.12 & $<12.1$ & -0.9 & \\
\hline J2020.8+3649 & PSR & 305.22 & 36.83 & 75.18 & 0.13 & $108.3 \pm 8.7$ & 12.4 & MGRO J2019+37 \\
\hline $\mathrm{J} 2021.5+4026$ & PSR & 305.40 & 40.44 & 78.23 & 2.07 & $35.8 \pm 8.5$ & 4.2 & \\
\hline $\mathrm{J} 2027.5+3334$ & & 306.88 & 33.57 & 73.30 & -2.85 & $<16.0$ & -0.2 & \\
\hline $\mathrm{J} 2032.2+4122$ & PSR & 308.06 & 41.38 & 80.16 & 0.98 & $63.3 \pm 8.3$ & 7.6 & $\begin{array}{c}\text { TEV 2032+41 } \\
\text { MGRO J2031+41 }\end{array}$ \\
\hline $\mathrm{J} 2055.5+2540$ & & 313.89 & 25.67 & 70.66 & -12.47 & $<17.6$ & -0.0 & \\
\hline $\mathrm{J} 2110.8+4608$ & & 317.70 & 46.14 & 88.26 & -1.35 & $<24.1$ & 1.1 & \\
\hline $\mathrm{J} 2214.8+3002$ & & 333.70 & 30.05 & 86.91 & -21.66 & $<20.7$ & 0.6 & \\
\hline $\mathrm{J} 2229.0+6114$ & PSR & 337.26 & 61.24 & 106.64 & 2.96 & $70.9 \pm 10.8$ & 6.6 & MGRO C4 \\
\hline $\mathrm{J} 2302.9+4443$ & & 345.75 & 44.72 & 103.44 & -14.00 & $<13.2$ & -0.6 & \\
\hline
\end{tabular}

Notes. The source identity in the 0FGL catalog is given with the source location in celestial and galactic coordinates. We give the measured flux for all sources above $3 \sigma$ at a characteristic median energy of $35 \mathrm{TeV}$. The $2 \sigma$ upper limits are given for other sources. The statistical significance and nearby TeV associations are noted.

a The BSL association with a known SNR is based on similar location.

the hypothesized spectrum. The median energy depends on declination and varies between 32 and $46 \mathrm{TeV}$ for $\delta$ in the range of $10^{\circ}-60^{\circ}$. At a declination of $-5^{\circ}$, the median energy of the hypothesized spectrum is $90 \mathrm{TeV}$. We quote the flux for all sources above $3 \sigma$ at a representative value of $35 \mathrm{TeV}$. It should be noted that the median energy used is for the assumed spectrum and not experimentally measured. In particular, a source may in fact cut off before $35 \mathrm{TeV}$ (the Crab for example) and our analysis would still report a flux at $35 \mathrm{TeV}$. The energy spectrum of each reported source is the subject of a paper in preparation.

The results of this search are summarized in Table 1. Of the 34 targets, 14 have a significance greater than $3 \sigma$. Six of these are associated with sources or candidates from the first Milagro survey of the Galactic plane (Abdo et al. 2007b). The Crab, MGRO 2019+37, MGRO 1908+06, MGRO 2031+41, and Milagro candidates C3 (likely associated with Geminga), and $\mathrm{C} 4$ (likely associated with the Boomerang pulsar wind nebula (PWN)) are all near LAT GeV sources. In the Milagro data set, the $3 \sigma-5 \sigma$ observations are fairly marginal because they cannot be convincingly discerned from background when statistical penalties for searching the entire sky are taken into account. However, with LAT points as a trigger for the search, the statistical penalties are reduced. The probability of a single $3 \sigma$ false positive in 34 samples of pure background is only $\sim 4.4 \%$. The probability of 4 or more excesses at or above $3 \sigma$ in 34 trials is $\sim 1.5 \times 10^{-7}$. It is very likely that most of our $3 \sigma$ excesses are due to multi-TeV emission. ${ }^{19}$ We, therefore, see strong evidence for multi-TeV emission associated with Galactic LAT BSL sources as a class, even if individual sources are not strong enough to be definitively detected by Milagro.

There is some contribution to these measurements from the Galactic diffuse emission, but that contribution is small. We can make a conservative estimate by taking the Milagro

\footnotetext{
19 Alternatively, using the False Discover Rate method (Miller et al. 2001; Hopkins et al. 2002) and requiring an estimate of $1 \%$ of the members of the selected candidates to be a false discovery, gives the same list of candidates. Changing the contamination fraction criterion from 0.01 to 0.001 (or to 0.1 ), would have included one fewer (or three more) sources, respectively.
} 

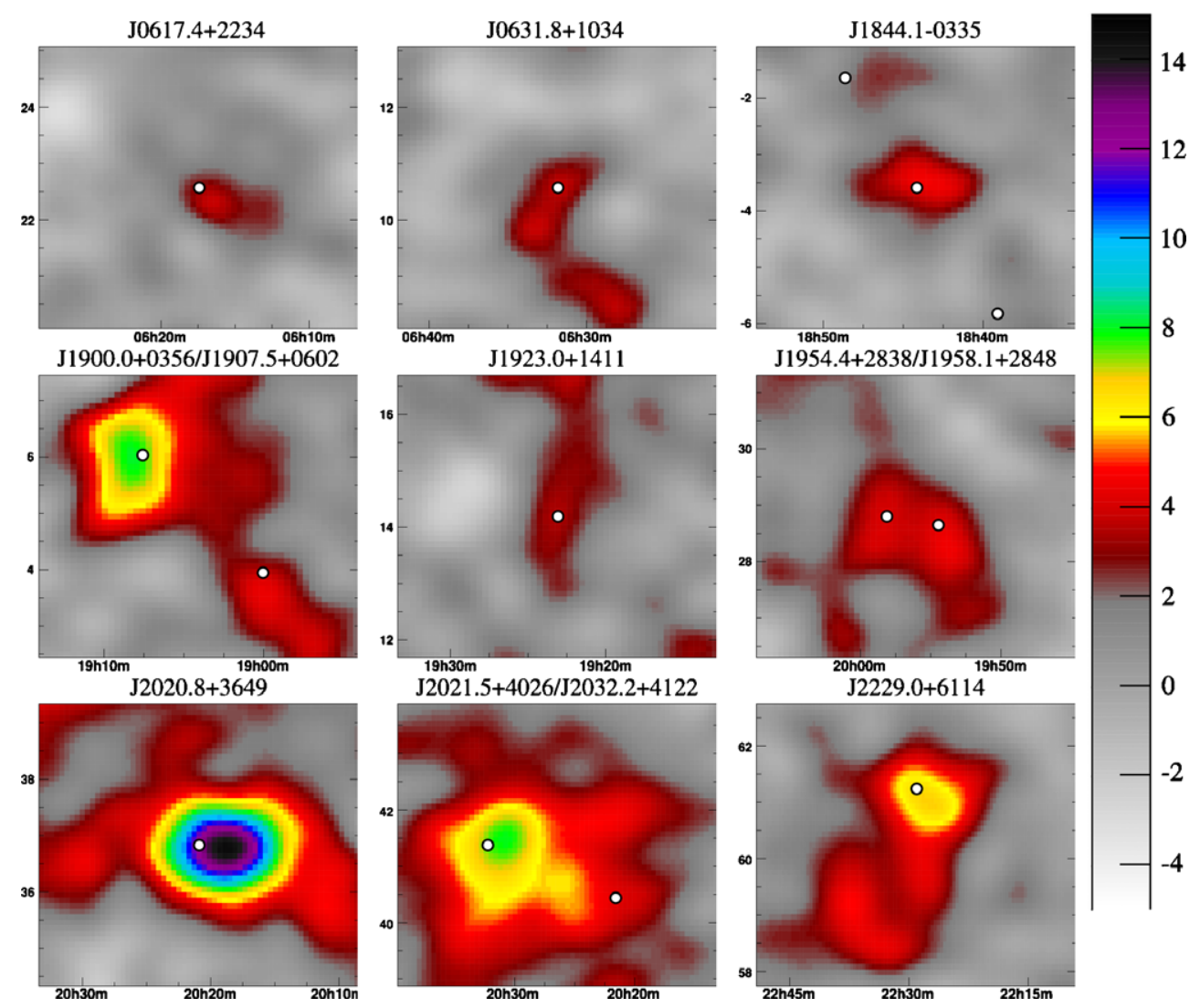

Figure 1. $3 \sigma$ sources from Table 1, omitting J0634.0+1745 (shown in Figure 2) and the Crab. Each frame shows a $5^{\circ} \times 5^{\circ}$ region with the LAT source positions indicated by white dots. The error on the Fermi source locations is quite small on this scale, typically between 0.1 and 0.2 deg, depending on the source. The data have been smoothed by a Gaussian of width varying between 0.4 and 1.0, depending on the expected angular resolution of events. Horizontal axes show right ascension and vertical axes show declination. The colors indicate the statistical significance in standard deviations.

measurement of the diffuse emission (Abdo et al. 2008) at its highest value, in the inner Galaxy $\left(30^{\circ}<l<65^{\circ},|b|<2^{\circ}\right)$. Using this value, we expect $5.3 \times 10^{-17} \mathrm{TeV}^{-1} \mathrm{~s}^{-1} \mathrm{~cm}^{-2}$ in a $1^{\circ}$ bin at $35 \mathrm{TeV}$, which is only about $\sim 15 \%$ contamination for the weakest sources in Table 1. The GALPROP conventional model, for comparison, would only constitute $\sim 3 \%$ contamination. The contamination is likely lower than suggested by the Milagro measurement because of unresolved sources, such as many of the sources from Table 1. It has even been suggested (Casanova \& Dingus 2008) that most of the Milagro diffuse measurement could be due to unresolved sources. Finally, the Fermi points observed at $3 \sigma$ in the Milagro data occur near local maxima in the Milagro data. In contrast, the diffuse emission is expected to vary slowly across the Galaxy.

\section{DISCUSSION}

From this analysis, it appears quite common for Galactic $100 \mathrm{MeV}$ to $100 \mathrm{GeV}$ sources to have associated multi-TeV emission. This association is notable for pulsars, where 9 of the 16 pulsars from the BSL are on our list of likely multi$\mathrm{TeV}$ emitters. The pulsars in the BSL which have less than $3 \sigma$ significance in Milagro data tend to lie off the Galactic plane. The pulsars off the plane are typically older, having traveled far from their origin after the kick they received from the initial asymmetric supernova (Gunn \& Ostriker 1970). Of the SNR sources on the list, we see three of five. Interestingly, we see only 2 of the 12 unidentified sources. These unidentified sources may be extragalactic and not visible with this analysis which was optimized for multi- $\mathrm{TeV}$ emission.
Figures 1 and 2 show the regions in the Milagro data around the indicated LAT sources. Eight of the 13 sources are associated with previously reported $>\mathrm{TeV}$ sources or candidates.

OFGL J0534.6+2201 is the young Crab Pulsar. Its associated $\mathrm{PWN}$ is a standard reference source in $\mathrm{TeV}$ astronomy.

OFGL J0617.4+2234 is associated with SNR IC443, which is interacting with a nearby large molecular cloud. An associated $\mathrm{X}$-ray feature has been interpreted as a PWN (Olbert et al. 2001), implying the existence of a pulsar, but the no pulsed emission has yet been detected. IC443 was first reported above $1 \mathrm{TeV}$ by MAGIC (Albert et al. 2007) and later confirmed by VERITAS (Humensky et al. 2008). The flux reported in Table 1 is somewhat higher than the flux predicted by extrapolating the MAGIC fit, but is roughly consistent after allowing for the extremes of the statistical and systematic errors of the two measurements.

OFGL J0634.0+1745 is the Geminga pulsar. Geminga is a relatively old (342 kyr) but very near (250 pc) pulsar (Manchester et al. 2005; Halpern \& Holt 1992; Faherty et al. 2007). It is the most significant Fermi-LAT source in the northern sky, but emission over $1 \mathrm{TeV}$ has only been reported by Milagro as candidate $\mathrm{C} 3$ with too low a significance to be classified as a definitive detection. Milagro observes an emission region that is extended by several degrees as shown in Figure 2. The significance reported in Table 1 has been computed assuming point-source emission, but if we instead assume that the source is due to emission from an extended region and convolve a $1^{\circ}$ Gaussian with the energy-dependent point-spread function, the significance at the location of 0FGL J0634.0+1745 increases to $6.3 \sigma$. The local maximum of the Milagro excess is at R.A. $=6^{\mathrm{h}} 32^{\mathrm{m}} 28^{\mathrm{s}}$, 

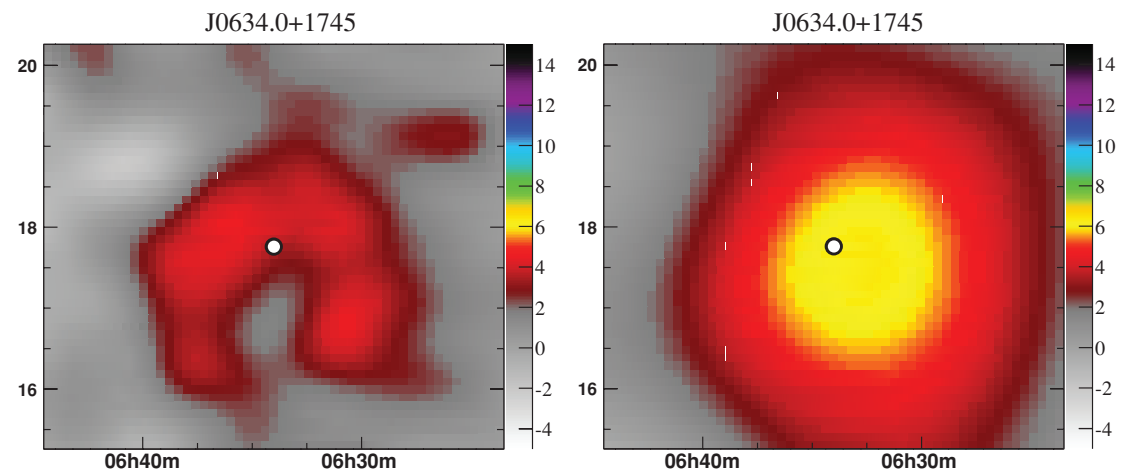

Figure 2. Significance of the Milagro data in a $5^{\circ} \times 5^{\circ}$ region around Fermi source J0634.0+1745 the Geminga pulsar. The location of the Fermi source is identified by a white dot. The figure on the left shows the significance map after smoothing by the Milagro point-spread function. The figure on the right shows the same region smoothed by an additional $1^{\circ}$ Gaussian in order for an extended emission region. The color scale shows the statistical significance.

decl. $=17^{\circ} 22^{\mathrm{m}}$. Given the high significance, we regard this as a definitive detection of extended emission from Geminga. A spatial Gaussian fit to the data yields a region with a standard deviation of $1.30 \pm 0.20$. For comparison, the analogous fit for the Crab, which is effectively a point source, has a $\sigma$ of 0.6 This suggests that the full width at half-maximum of the region of emission in the vicinity of Geminga is $2.6_{-0.9}^{+0.7}$, after accounting for the point-spread function. The large extent (implying an emission region of some $7-15$ pc extent) is likely due to the nearness of the source and may arise from a pulsar-driven wind; it is consistent with H.E.S.S. observation of more distant PWN with an angular size of $\sim 10 \mathrm{pc}$. This may also explain why the source has not yet been observed by Imaging Atmospheric Cherenkov Telescopes (Maier et al. 2008).

OFGL J1907.5+0602 is associated with MGRO J1908+06 (Abdo et al. 2007b). This pulsar was discovered by the LAT and is also coincident with AGILE source 1AGL J1908+0613 (Pittori et al. 2009) and EGRET source GEV J1907+557 (Lamb \& Macomb 1997). The multi-TeV emission was first reported by Milagro. H.E.S.S. both confirmed the Milagro detection and was also able to identify this source as extended by $0.21_{-0.05}^{+0.07}$ (Djannati-Atai et al. 2008). The peak of the Milagro detection occurs at R.A. $=19^{\mathrm{h}} 6^{\mathrm{m}} 44^{\mathrm{s}}$, decl. $=5^{\circ} 50^{\mathrm{m}}$ with a $1 \sigma$ error circle of 0.27 and a local peak significance of $8.1 \sigma$. The peak of the Milagro emission is 0.3 from the pulsar, but consistent with the pulsar's location within the measurement error.

OFGL J1923.0+1411 is associated with SNR G49.2-0.7 (W51) which is in a star-forming region and near molecular clouds. Recently, a $>\mathrm{TeV}$ source, H.E.S.S. J1923+141 (Feinstein et al. 2009), has been detected which is spatially extended and coincident with the Fermi source.

OFGL J2020.8+3649 is associated with MGRO J2019+37. This is the most significant source in the Milagro data set apart from the Crab. The young central pulsar has a period of $104 \mathrm{~ms}$ and an estimated age of $17.2 \mathrm{kyr}$. This source was also detected by AGILE and EGRET. It was AGILE that first identified the GeV pulsations (Halpern et al. 2008) and that discovery was confirmed with Fermi data. The peak of the flux measured by Milagro is at R.A. $=20^{\mathrm{h}} 18^{\mathrm{m}} 43^{\mathrm{s}}$ decl. $=36^{\circ} 42^{\mathrm{m}}$ with a $0.091 \sigma$ error circle. The position of the excess is $\sim 0.3$ from the pulsar.

OFGL J2032.2+4122 is a LAT identified pulsar that is spatially coincident with the HEGRA source J2032+41 (Aharonian et al. 2002), MGRO J2031+41, and the MAGIC source J2032+4130 (Albert et al. 2008). The Milagro source was reported (Abdo et al. 2007b) with an extent of $3^{\circ}$, but it appears that the Milagro extended source may be due to two or more overlapping sources with a potential additional diffuse contri- bution from the highly emissive Cygnus region. The location of the Milagro peak is R.A. $=20^{\mathrm{h}} 31^{\mathrm{m}} 43^{\mathrm{s}}$ and decl. $=40^{\circ} 40^{\mathrm{m}}$ with a statistical error of 0.3 .

OFGL J2229.0+6114 is coincident with the radio pulsar J2229+6114 which has been previously associated (Halpern et al. 2001) with the EGRET source 3EG J2227+6122. The period of this pulsar is $52 \mathrm{~ms}$, its distance is $0.8 \mathrm{kpc}$ (Kothes et al. 2001), and the age is estimated to be $10.5 \mathrm{kyr}$, and $\dot{E}$ is $2.2 \times 10^{37} \mathrm{erg} \mathrm{s}^{-1}$ (Manchester et al. 2005; Halpern et al. 2001). Milagro detects a $6.6 \sigma$ excess at the position of the pulsar and a local maximum of $6.8 \sigma$. The peak of the Milagro excess is R.A. $=22^{\mathrm{h}} 28^{\mathrm{m}} 17^{\mathrm{s}}$ decl. $=60^{\circ} 29^{\mathrm{m}}$ with a statistical position error of 0.36 . This source was reported as candidate $\mathrm{C} 4$ by Milagro in Abdo et al. (2007b). With the additional data and improved analysis presented here, this source is elevated to a high-confidence detection. Milagro also identifies this source as clearly not a point source, with a long extension to the south. ${ }^{20}$

The remaining five objects with greater than $3 \sigma$ excess in the Milagro data have not been previously detected above $1 \mathrm{TeV}$ energies.

OFGL J0631.8+1034 is the radio pulsar $\mathrm{J} 0631+10$ (Zepka et al. 1996). This pulsar has a period of $288 \mathrm{~ms}$ and an estimated age of $43.6 \mathrm{kyr}$, a distance of $6.55 \mathrm{kpc}$, and $\dot{E}$ of $1.7 \times 10^{35} \mathrm{erg}$ $\mathrm{s}^{-1}$ (Manchester et al. 2005). The VERITAS upper limit for this region is $1.3 \%$ of the Crab (Maier et al. 2008).

OFGL J1844.1-0335 is unassociated with any known source. It is an interesting source because it occurs at a declination at the edge of Milagro's sensitivity and, if the Milagro observation is real, it is extremely bright above $1 \mathrm{TeV}$. It is in the region of the Galactic plane surveyed by H.E.S.S. (Aharonian et al. 2006) but was not detected. To account for the H.E.S.S. nondetection, the source would have to be extended or have a very hard spectrum extending to high energy.

OFGL J1900.0+0356 has no known associations.

OFGL J1954.4+2838 is coincident with SNR G65.1+0.6 which has been associated with PSR 1957+2831 (Tian \& Leahy 2006).

OFGL J1958.1+2848 is a LAT-discovered pulsar that is associated with the EGRET source 3EG J1958+2909 (Hartman et al. 1999).

\footnotetext{
20 Note added in press: the Fermi-LAT collaboration has completed a paper announcing the discovery of a new pulsar-not included in the BSL-with the current best position of R.A. $=339.561$, decl. $=59.080$ (Fermi-LAT Collaboration 2009, http://www.sciencemag.org/cgi/content/abstract/ $1175558 \mathrm{v} 2)$. Milagro observes a $4.7 \sigma$ excess at the location of the pulsar. It may be that the large size of the multi-TeV emission associated with 0FGL $\mathrm{J} 2229.0+6114$ is in fact due to these two nearby sources.
} 

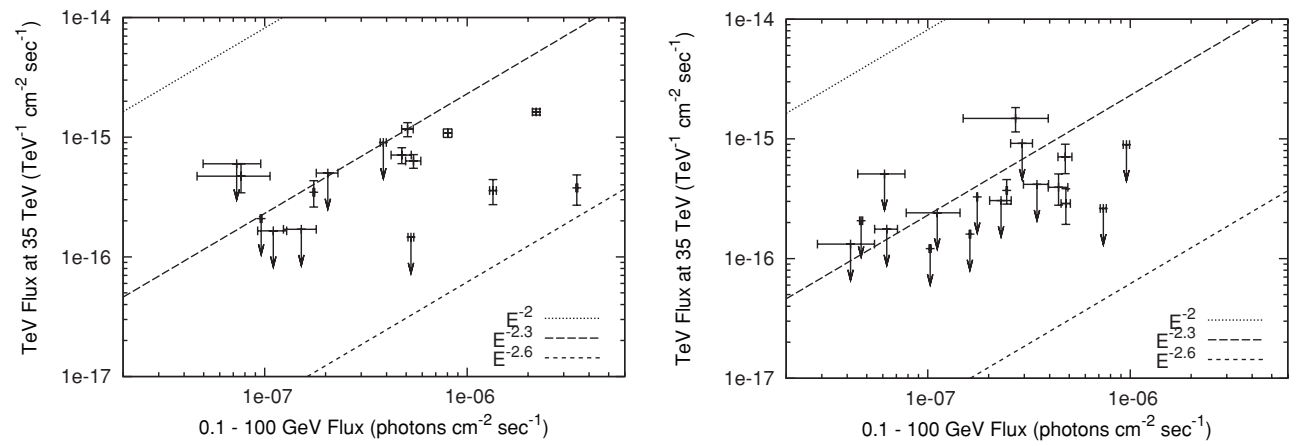

Figure 3. Flux estimates and upper limits for the 34 Fermi sources. The horizontal axis quotes the integral Fermi flux from $100 \mathrm{MeV}$ to $100 \mathrm{GeV}$ and the vertical axis gives the Milagro flux or upper limit at $35 \mathrm{TeV}$. Lines are shown with the extrapolation of the Fermi flux to Milagro energies, assuming an $E^{-2.0}, E^{-2.3}$, and $E^{-2.6}$ spectrum. The left panel shows the results for the 16 pulsars and the right panel shows the results for the remaining 18 sources.

OFGL J2021.5+4026 is a LAT-discovered pulsar that is coincident with the gamma-Cygni SNR. This source is located in the Cygnus region that is detected by Milagro as having a broad extended excess.

The relationship between the Fermi and Milagro source fluxes and upper limits for these 34 sources is shown in Figure 3. The BSL values for the integral flux are shown with the Milagro measurements of the differential flux at $35 \mathrm{TeV}$. The $35 \mathrm{TeV}$ fluxes are roughly correlated with the measurements between $100 \mathrm{MeV}$ and $100 \mathrm{GeV}$ but the correlation is not strong, with a correlation coefficient of the $3 \sigma$ points in log space of only 0.2 . One possible explanation for the pulsar variation is that the pulsed emission is expected to be beamed (and thus viewingangle dependent) and the unpulsed multi-TeV emission is likely unbeamed (Gaensler \& Slane 2006). The spectrum that connects the Milagro flux to the Fermi flux is universally softer than 2.0 and closer to 2.3, depending on the source.

We have found that the population of Fermi sources observed at or above $3 \sigma$ by Milagro is dominated by pulsars and/or their associated PWN. Of the four high-confidence Milagro detections associated with pulsars of known periodicity and distance, three (namely, J0534.6+2201, J0634.0+1745, and J2229.0+6114) have $\dot{E} / d^{2}$ above $10^{35} \mathrm{erg} \mathrm{s}^{-1} \mathrm{kpc}^{-2}$ where $\dot{E}$ is the spin-down luminosity and $d$ is the distance to the pulsar. The distance on the fourth $(\mathbf{J} 2020.8+3649)$ is uncertain. Using the 3-4 kpc distance implied by X-ray measurements (Van Etten et al. 2008) rather than the $12 \mathrm{kpc}$ measurement implied by the pulsar's dispersion measure (DM), it too has $\dot{E} / d^{2}$ above $10^{35}$ $\mathrm{erg} \mathrm{s}^{-1} \mathrm{kpc}^{-2}$. A similar association with high $\dot{E} / d^{2}$ pulsars is reported by H.E.S.S. (Carrigan et al. 2008). Since the pulsed emission is beamed and the PWN is not, all high $\dot{E} / d^{2}$ are possible candidates for multi- $\mathrm{TeV}$ emission. We have searched the ATNF pulsar database (Manchester et al. 2005) for northernhemisphere pulsars with a high $\dot{E} / d^{2}$, which were not reported in the Fermi BSL. Of the 25 highest $\dot{E} / d^{2}$ pulsars, there are 10 in the northern hemisphere and 5 not identified as $\mathrm{GeV}$ sources by Fermi. These five are J0205+6449, J0659+1414, $\mathrm{J} 1930+1852, \mathrm{~J} 1913+1011$, and $\mathrm{J} 1740+1000$. Of these, the largest statistical significance was 3.3 standard deviations (PSR $\mathrm{J} 1930+1852$ ), not significant enough to claim this as new source of multi-TeV gamma rays (though follow-up observations are warranted).

Finally, it is interesting to note that of the sources published in the Milagro survey of the Galactic plane (Abdo et al. 2007b), all four sources and two of the four source candidates are now strongly associated with pulsars, suggesting that most of the Milagro sources are multi-TeV PWN. We also note the high efficiency with which $\mathrm{MeV}$ to $\mathrm{GeV}$ pulsars are observed above $1 \mathrm{TeV}$, and a qualitative picture is emerging where the typical
Galactic multi-TeV source is a PWN associated with a $\mathrm{MeV}$ to $\mathrm{GeV}$ pulsar.

We gratefully acknowledge Scott Delay and Michael Schneider for their dedicated efforts in the construction and maintenance of the Milagro experiment. This work has been supported by the National Science Foundation (under grants PHY-0245234, -0302000, -0400424, -0504201, -0601080, and ATM-0002744), the US Department of Energy (Office of HighEnergy Physics and Office of Nuclear Physics), Los Alamos National Laboratory, the University of California, and the Institute of Geophysics and Planetary Physics.

\section{REFERENCES}

Abdo, A. A., et al. 2007a, ApJ, 658, L33

Abdo, A. A., et al. 2007b, ApJ, 664, L91

Abdo, A. A., et al. 2008, ApJ, 688, 1078

Abdo, A. A., et al. 2009, ApJS, 183, 46

Aharonian, F., et al. 2002, A\&A, 393, L37

Aharonian, F., et al. 2006, ApJ, 636, 777

Albert, J., et al. 2007, ApJ, 664, L87

Albert, J., et al. 2008, ApJ, 675, L25

Carrigan, S., et al. 2008, in Proc. 30th Int. Cosmic Ray Conf., 2, ed. R. Caballero, et al. (Mexico City: Universidad Nacional Autónoma de México), 659

Casanova, S., \& Dingus, B. L. 2008, Astropart. Phys., 29, 63

Djannati-Atai, A., et al. 2008, in Proc. 30th Int. Cosmic Ray Conf.,2, ed. R. Caballero, et al. (Mexico City: Universidad Nacional Autónoma de México), 831

Faherty, J., Walter, F. M., \& Anderson, J. 2007, Ap\&SS, 308, 225

Feinstein, F., et al. 2009, in AIP Conf. Proc. 1112, Science with the New Generation of High Energy Gamma-ray Experiments, ed. D. Bastieri \& R. Rando (Melville, NY: AIP), 54

Gaensler, B. M., \& Slane, P. O. 2006, ARA\&A, 44, 17

Gunn, J. E., \& Ostriker, J. P. 1970, ApJ, 160, 979

Halpern, J. P., \& Holt, S. S. 1992, Nature, 357, 222

Halpern, J. P., et al. 2001, ApJ, 552, L125

Halpern, J. P., et al. 2008, ApJ, 688, L33

Hartman, R. C., et al. 1999, ApJS, 123, 79

Hopkins, A. M., Miller, C. J., Connolly, A. J., Genovese, C., Nichol, R. C., \& Wasserman, L. 2002, AJ, 123, 1086

Humensky, T. B., et al. 2008, in Proc. AIP Conf. Ser. 1085, High Energy Gammaray Astronomy, ed. F. A. Aharonian, W. Hofmann, \& F. Rieger (Melville, NY: AIP), 357

Kothes, R., Uyaniker, B., \& Pineault, S. 2001, ApJ, 560, 236

Lamb, R. C., \& Macomb, D. J. 1997, ApJ, 488, 872

Maier, G., et al. 2008, in AIP Conf. Ser. 1085, High Energy Gamma-ray Astronomy, ed. F. A. Aharonian, W. Hofmann, \& F. Rieger (Melville, NY: AIP), 187

Manchester, R. N., Hobbs, G. B., Teoh, A., \& Hobbs, M. 2005, AJ, 129, 1993

Miller, C. J., et al. 2001, AJ, 122, 3492

Olbert, C. M., Clearfield, C. R., Williams, N. E., Keohane, J. W., \& Frail, D. A. 2001, ApJ, 554, L205

Pittori, C., et al. 2009, A\&A, submitted (arXiv:0902.2959)

Tian, W. W., \& Leahy, D. A. 2006, A\&A, 455, 1053

Van Etten, A., Romani, R. W., \& Ng, C.-Y. 2008, ApJ, 680, 1417

Zepka, A., Cordes, J. M., Wasserman, I., \& Lundgren, S. C. 1996, ApJ, 456 , 305 